\title{
Efficient gating of epitaxial boron nitride monolayers by substrate functionalization
}

\author{
A. Fedorov, ${ }^{1,2,3,{ }^{*}}$ C. S. Praveen,,${ }^{4,}$ N. I. Verbitskiy,,${ }^{1,5,6}$ D. Haberer, ${ }^{7}$ D. Usachov, ${ }^{3}$ D. V. Vyalikh, ${ }^{3,8}$ A. Nefedov, ${ }^{9}$ C. Wöll, ${ }^{9}$ \\ L. Petaccia, ${ }^{10}$ S. Piccinin, ${ }^{4}$ H. Sachdev, ${ }^{11}$ M. Knupfer, ${ }^{2}$ B. Büchner, ${ }^{2}$ S. Fabris, ${ }^{4, \dagger}$ and A. Grüneis ${ }^{1, \ddagger}$ \\ ${ }^{1}$ II. Physikalisches Institut, Universität zu Köln, Zülpicher Strasse 77, 50937 Köln, Germany \\ ${ }^{2}$ Institute for Solid State Research, IFW Dresden, P.O. Box 270116, D-01171 Dresden, Germany \\ ${ }^{3}$ St. Petersburg State University, St. Petersburg 198504, Russia \\ ${ }^{4}$ Theory@Elettra Group, CNR-IOM DEMOCRITOS and SISSA, via Bonomea 265, 34136 Trieste, Italy \\ ${ }^{5}$ Faculty of Physics, University of Vienna, Strudlhofgasse 4, A-1090 Vienna, Austria \\ ${ }^{6}$ Department of Materials Science, Moscow State University, Leninskiye Gory 1/3, 119992 Moscow, Russia \\ ${ }^{7}$ Department of Physics, University of California at Berkeley, Berkeley, California 94720, USA \\ ${ }^{8}$ Institute of Solid State Physics, Dresden University of Technology, D-01062 Dresden, Germany \\ ${ }^{9}$ Institute für Funktionelle Grenzflächen (IFG), Karlsruher Institut für Technologie (KIT), Hermann-von-Helmoltz-Platz 1, \\ D-76344 Eggenstein-Leopoldshafen, Germany \\ ${ }^{10}$ Elettra Sincrotrone Trieste, Strada Statale $14 \mathrm{~km}$ 163.5, 34149 Trieste, Italy \\ ${ }^{11}$ Max-Planck-Institut für Polymerforschung, Ackermannweg 10, D-55128 Mainz, Germany
}

(Received 15 May 2015; revised manuscript received 4 August 2015; published 28 September 2015)

\begin{abstract}
Insulating hexagonal boron nitride monolayers $(h \mathrm{BN})$ are best known for being resistant to chemical functionalization. This property makes $h \mathrm{BN}$ an excellent substrate for graphene heterostructures, but limits its application as an active element in nanoelectronics where tunable electronic properties are needed. Moreover, the two-dimensional-materials' community wishes to learn more about the adsorption and intercalation characteristics of alkali metals on $h \mathrm{BN}$, which have direct relevance to several electrochemistry experiments that are envisioned with layered materials. Here we provide results on ionic functionalization of $h \mathrm{BN} / \mathrm{metal}$ interfaces with $\mathrm{K}$ and $\mathrm{Li}$ dopants. By combining angle-resolved photoemission spectroscopy (ARPES), $\mathrm{x}$-ray photoelectron spectroscopy, and density functional theory calculations, we show that the metallic substrate readily ionizes the alkali dopants and exposes $h \mathrm{BN}$ to large electric fields and band-energy shifts. In particular, if $h \mathrm{BN}$ is in between the negatively charged substrate and the positive alkali ion, this allows us to directly study, using ARPES, the effects of large electric fields on the electron energy bands of $h \mathrm{BN}$.
\end{abstract}

DOI: 10.1103/PhysRevB.92.125440

PACS number(s): 73.22.-f

\section{INTRODUCTION}

Hexagonal boron nitride $(h \mathrm{BN})$, a structural analog to graphene, has been attracting considerable research attention due to its outstanding mechanical properties, its potential for surface chemistry [1], and its potential applications in far-ultraviolet (far-UV) optoelectronic devices [2,3] and twodimensional (2D) heterostructures comprising graphene and $h \mathrm{BN}[4,5]$. First results on chemical functionalization of $h \mathrm{BN}$ have also been reported by trapping atoms or molecules on the dipole rings of epitaxial boron nitride [1]. The ionicity of the B-N bond induces a wide band gap in $h \mathrm{BN}$ with a direct optical transition for bulk $h \mathrm{BN}$ of about $5.9 \mathrm{eV}[3,6,7]$. The lack of electronic states at or close to the Fermi level also limits the chemical activity of $h \mathrm{BN}$. In contrast to carbon materials, where ionic [8], covalent [9], and substitutional [10] doping have been successfully carried out, the interaction of potassium with bulk $h \mathrm{BN}$ does not result in charge transfer to the electronic states of $h \mathrm{BN}$ [11] owing to the large energy band gap. This insulating and chemically inert behavior is exploited in novel $2 \mathrm{D}$ devices which use $h \mathrm{BN}$ as a substrate for graphene in order to attain high electronic mobilities. In such epitaxial $h \mathrm{BN} /$ graphene heterostructures, a small gap in

\footnotetext{
*Authors contributed equally to this work.

†fabris@democritos.it

†rueneis@ph2.uni-koeln.de
}

graphene is predicted [12] and the highest mobilities known for graphene are achieved [13]. But the lack of response to chemical functionalization severely limits the usefulness of $h \mathrm{BN}$ as an active element, which has prompted the search for alternative methods to manipulate its electronic properties. In particular, the application of large electric fields leads to a giant Stark effect, which was theoretically predicted for $h \mathrm{BN}$ nanotubes [14] and $h \mathrm{BN}$ nanoribbons [15]. Experimental evidence of this, albeit only at the single-molecule level, was gathered by scanning tunneling spectroscopy (STS) measurements for tip-induced electric fields in the vicinity of a $h \mathrm{BN}$ nanotube [16]. Electric fields applied to $h \mathrm{BN}$ tubes with a controlled defect concentration are also the basis for a recently predicted gate-tunable light-emitting device [17]. Field effect transistors (FETs) based on $h \mathrm{BN}$ nanotubes exhibit a robust ON/OFF ratio [18]. The parent compounds of these systems are $h \mathrm{BN}$ monolayers [19-21] and $h \mathrm{BN}$ nanomeshes [22], which can be grown epitaxially in high crystallinity in areas exceeding $1 \times 1 \mathrm{~cm}$ by chemical-vapor deposition (CVD) on metals. Recently, $h \mathrm{BN}$ was found to support space-charge-limited current, which is promising for UV-light-emitting diodes and laser diodes [23]. Despite the simple structure and the theoretical progress in understanding of the $h \mathrm{BN}$ band structure, little is known about the effects of gating and electric fields on their electronic properties. This is a direct result of the fact that in order to apply a homogeneous electronic gating, the $h \mathrm{BN}$ monolayer must be transferred from the catalytic metal substrate to an insulator 
and supplied with electric contacts. The resulting devices are usually too small [23] to be probed by angle-resolved photoemission spectroscopy (ARPES), which relies on large (i.e., in the $\mathrm{mm}$ range) single-crystalline materials.

Here we take a different approach by exposing $h \mathrm{BN}$ to the large potentials and electric fields generated by an intercalated or adsorbed layer of ionized alkali metal. This not only relieves us from the task of transferring $h \mathrm{BN}$ but also retains the large size and high crystallinity of the $h \mathrm{BN}$ layer, which allows us to directly probe the effects of the electric field on the electron energy band structure by ARPES. In addition, we employ x-ray photoemission spectroscopy (XPS) and near-edge $\mathrm{X}$-ray absorption fine-structure spectroscopy (NEXAFS). These methods allow us to study the interface structure of the dopant/hBN system, i.e., whether the dopant preferably is above or under $h \mathrm{BN}$, and to probe the unoccupied states, respectively. The crucial point in this approach is that $h \mathrm{BN}$ is on a Au substrate that readily ionizes the deposited alkali atoms, which is somewhat similar for doped graphene on metal substrates, where ARPES and density functional theory (DFT) have shown that a part of the electron charge is transferred to the metal $[8,24]$. The present case is different from the graphene case, in the sense that graphene accepts a large fraction of the alkali atom's charge while $h \mathrm{BN}$ does not. Our work, therefore, constitutes an experimental realization of chemical functionalization of an $h \mathrm{BN} /$ metal interface using alkali metals and its direct measurement using ARPES. The possibility to tune the electronic properties of $h \mathrm{BN}$ monolayers is also highly relevant to the field of novel $h \mathrm{BN}$-graphene heterostructures. We purposefully engineer $h \mathrm{BN} /$ potassium/Au and lithium $/ h \mathrm{BN} / \mathrm{Au}$ structures. DFT calculations reproduce the ARPES data and are used for their interpretation: These simulations show a clear relationship between the energy shift of the $h \mathrm{BN}$ electron energy bands and the presence of interfacial potential differences and electric fields induced by the alkali metals.

\section{RESULTS}

Figure 1(a) depicts the XPS measurements that show the B1s and $\mathrm{N} 1 s$ core levels for pristine and alkali-metal functionalized $h \mathrm{BN}$. It is clear that both core levels exhibit a shift towards higher binding energy (BE) in the latter case. This behavior is similar to graphene and graphite [8,24]. However, as we will show in the following, it is not due to a charge transfer but interfacial electric fields that yield potential differences between the substrate and $h \mathrm{BN}$. We also observe a different shift for the $\mathrm{N} 1 s$ and $\mathrm{B} 1 s$ core levels. This can be ascribed to a combination of initial- and final-state effects originating from the charge reorganization at the $h \mathrm{BN}$ plane due to the dopant deposition.

It is notable that during all photoemission experiments, the $\mathrm{hBN}$ layer was not charging, i.e., the Au substrate forms a good electrical contact to hBN. As we will show later, this good contact is formed by a weak hybridization between hBN and $\mathrm{Au}$.

Let us consider the XPS measurements of the alkali-metal core levels in more detail. Figure 1(b) depicts the angledependent XPS spectra of both the K $2 p$ and Li1 $s$ core levels. The relative intensity ratio (normalized to the N1s core-level
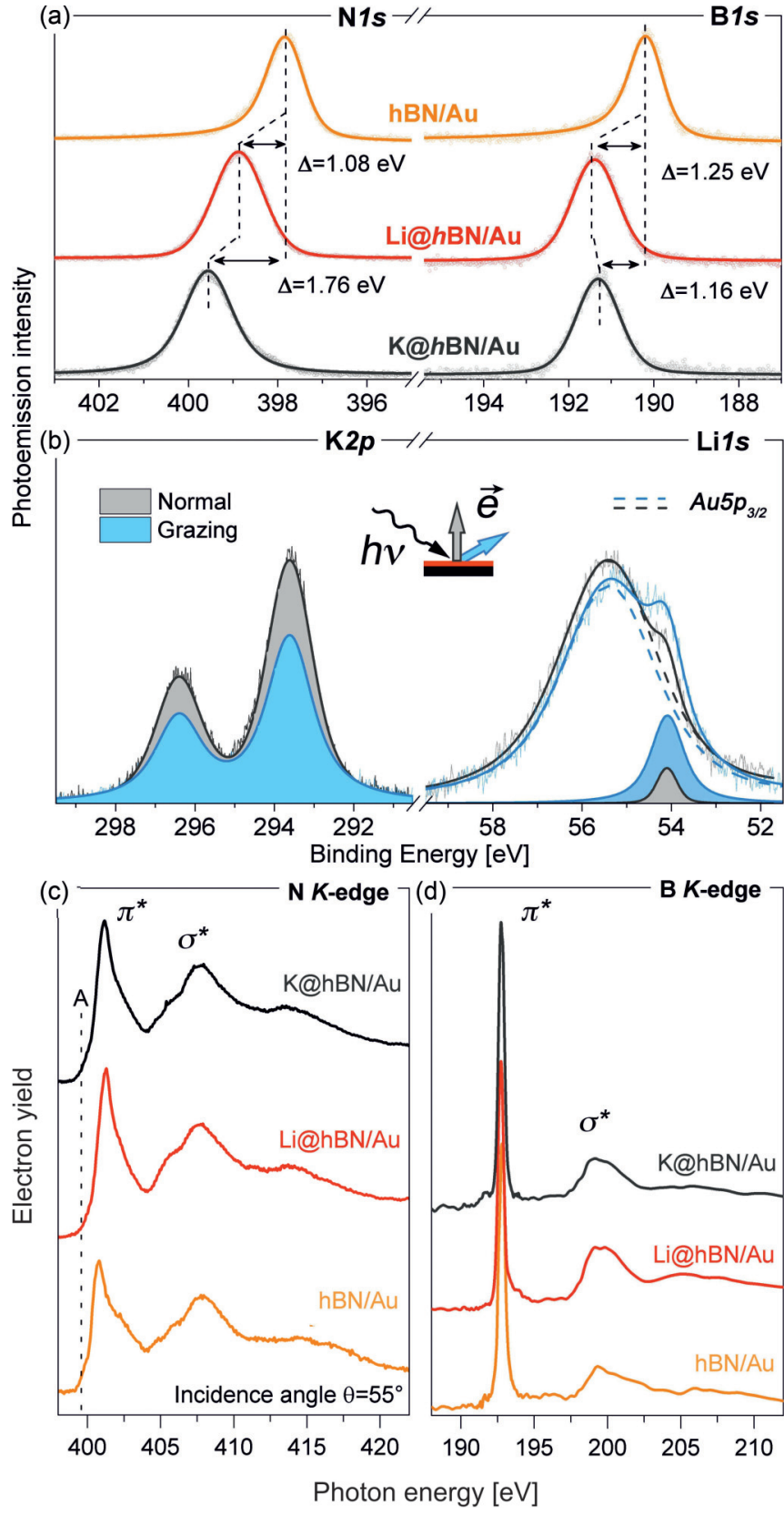

FIG. 1. (Color online) (a) $h \mathrm{BN}$ core-level spectra measured by XPS for pristine and alkali-metal-doped $h \mathrm{BN}$. (b) Normal and grazing emission core-level spectra of the alkali metals. (c),(d) NEXAFS spectra for the $\mathrm{N}$ and $\mathrm{B} K$ edges, respectively, for pristine and alkalimetal-doped $h \mathrm{BN}$.

intensity) indicates the preferred location of each species (either above or below the $h \mathrm{BN}$ plane) [24]. As a result, we obtain a preference of $\mathrm{K}$ to intercalate in between $h \mathrm{BN}$ and $\mathrm{Au} / \mathrm{Ni}(111)$ and a preference of $\mathrm{Li}$ to adsorb on top of $h \mathrm{BN}$. We note that this behavior is identical to $\mathrm{K}$ and $\mathrm{Li}$ interaction with graphene on $\mathrm{Au} / \mathrm{Ni}(111)$ [24]. We speculate that the different behavior for various alkali metals stems from the preference of some of them to form surface alloys while others do not.

In order to probe the effects of the alkali ions on the conduction band of $h \mathrm{BN}$, we performed NEXAFS measurements. 
This technique probes the transitions from N1s and B1s core levels to the unoccupied $\pi^{*}$ and $\sigma^{*}$ states and gives information about the hybridization of the electronic states. No extra peaks appear in the NEXAFS spectrum upon doping, but for both, pristine and doped $h \mathrm{BN}$, a small shoulder is present in the $\mathrm{N} 1 s$ edge when compared to bulk $h \mathrm{BN}$, as reported earlier [25]. In the present case, this shoulder is much weaker since the interaction between $\mathrm{hBN}$ and $\mathrm{Au}$ is weaker than the interaction between $\mathrm{hBN}$ and $\mathrm{Ni}$. Nevertheless, it allows us to conclude that the $h \mathrm{BN}$ is weakly interacting with the substrate. This weak interaction is also evident from the ARPES data via a hybridization and is crucially important for the effects described below since it allows us to treat the metal- $h \mathrm{BN}$ interface as one system with a common Fermi energy and work function.

Let us focus now on the band structure modifications. In Fig. 2, the ARPES scan along the $\Gamma K$ high-symmetry direction in the 2D Brillouin zone (BZ) of $h \mathrm{BN}$ for pristine as well as Liand K-doped samples is presented [Figs. 2(a)-2(c)]. Similar to the core levels of $h \mathrm{BN}$, the $\pi$ band shifts down after alkali deposition. In the case of $\mathrm{K}$, the downshift is $2.77 \mathrm{eV}$, while for the Li case, it is $0.9 \mathrm{eV}$. The large downshift in the case of $\mathrm{K}$ doping brings the top of the $\pi$ band in the vicinity of the Au5d bands, allowing a weak hybridization which can be seen as a loss of photoemission intensity in the band-crossing regions in the top of the $\pi$ band [Fig. 2(d)]. Concomitant with the NEXAFS results, this small hybridization highlights that the $h \mathrm{BN} / \mathrm{Au}$ interface must be treated as one coupled, weakly interacting system. The anticrossing in the $\sigma$ bands of $h \mathrm{BN}$ is analyzed in Fig. 2(e). The energy-dispersion curves through the $K$ point in the region of the anticrossing are shown for pristine and doped $h \mathrm{BN}$ on $\mathrm{Au}$. It can be seen that for $\mathrm{Li}$ doping, the anticrossing persists, while $\mathrm{K}$ doping removes the anticrossing in the $\sigma$ bands at $K$. This behavior is consistent with the suggested position of $\mathrm{Li}$ on top of $h \mathrm{BN}$ and the intercalation of $\mathrm{K}$ in between the $h \mathrm{BN} / \mathrm{Au}$ interface. In the case of $\mathrm{K}$ intercalation, the $h \mathrm{BN}$ layer is lifted up and so the interaction of the in-plane $\sigma$ orbitals with the substrate is removed. Moreover, we observe a strong reduction of ARPES intensity at the Fermi level due to the $\mathrm{K}$ intercalation, which suppresses the Fermi-level photoemission intensity dominated by Au orbitals due to the smaller inelastic mean free path in the $\mathrm{K}$ layer. The fact that, after $\mathrm{K}$ intercalation, hybridization with $\mathrm{Au}$ appears in the $\pi$ bands despite the larger distance between $h \mathrm{BN}$ and Au can be understood by the matching energies of $\pi$ and Au5d levels and by the larger extent of the $\pi$ orbitals in the $z$ direction compared to the $\sigma$ orbitals which lie in the $h \mathrm{BN}$ plane. In the case of Li doping, the hybridization of states at the $\pi$-band maximum at the $K$ point and Au5d is not possible since their energies do not match.

We now turn to a discussion of the qualitative reason for the observed downshifts of the $h \mathrm{BN}$ electronic bands before a quantitative description using DFT. The driving force behind these observed changes is the interaction of the Au substrate with the alkali atom. This can lead to the combined effects of gating via a potential and electric fields acting on $h \mathrm{BN}$. In particular, since the $\mathrm{K}$ atoms are readily ionized by the $\mathrm{Au}$ substrate, $h \mathrm{BN}$ is exposed to an electric potential which is able to shift its electronic band structure. The same effect will occur for $\mathrm{Li} / h \mathrm{BN} / \mathrm{Au}$ but, due to its sandwichlike structure where
TABLE I. Energy shifts of the $\pi$ bands in the functionalized systems where the alkali atom $(\mathrm{A})$ is above $(\mathrm{A} / h \mathrm{BN} / \mathrm{Au})$ or below $(h \mathrm{BN} / \mathrm{A} / \mathrm{Au})$ the $h \mathrm{BN}$ sheet. The energy shifts are expressed in $\mathrm{eV}$ and are relative to the top of the $h \mathrm{BN} \pi$ band in the pristine $\mathrm{BN} / \mathrm{Au}$ system.

\begin{tabular}{lccc}
\hline \hline $\begin{array}{l}\text { Alkali atom } \\
\mathrm{A}\end{array}$ & $\begin{array}{l}\text { Above } h \mathrm{BN} \\
\mathrm{A} / h \mathrm{BN} / \mathrm{Sub}\end{array}$ & $\begin{array}{c}\text { Below } h \mathrm{BN} \\
h \mathrm{BN} / \mathrm{A} / \mathrm{Sub}\end{array}$ & Experiment \\
\hline $\mathrm{Li}$ & 1.80 & 2.15 & 0.9 \\
$\mathrm{~K}$ & 1.50 & 2.73 & 2.77 \\
\hline \hline
\end{tabular}

$h \mathrm{BN}$ is in between $\mathrm{Li}^{+}$and $\mathrm{Au}^{-}$, there is an additional electric field between the ionized $\mathrm{Li}$ atom and the Au surface present.

Performing DFT calculations, we find that the actual energy shift of the $h \mathrm{BN}$ bands depends on many factors, most notably the coverage of the $\mathrm{Li}$ and $\mathrm{K}$ dopants, as well as their position with respect to the $h \mathrm{BN}$ layer. The calculations were performed by assuming the same coverage of $1 / 8$ monolayer with respect to the honeycomb layer for both $\mathrm{Li}$ and $\mathrm{K}$. Although this choice may not represent the actual experimental conditions, it provides a valuable insight into the effects of the two dopants on the BN/Sub system, removing the effects of different dopant coverage. Concerning the position of the dopant, we consider different locations of the $\mathrm{Li}$ and $\mathrm{K}$ dopants at the interface, namely, above and below the $h \mathrm{BN}$ layer. We refer to these four cases as $\mathrm{Li} / h \mathrm{BN} / \mathrm{Sub}, h \mathrm{BN} / \mathrm{Li} / \mathrm{Sub}, \mathrm{K} / h \mathrm{BN} / \mathrm{Sub}$, and $h \mathrm{BN} / \mathrm{K} / \mathrm{Sub}$, where "Sub" indicates the Au/Ni substrate. The summarized results of the corresponding $\pi$-band energy shifts are shown in Table I: alkali atom intercalation always yields the largest shifts. This is because the interaction with the metal substrate facilitates the charge transfer, which consistently leads to the charge of the intercalated ions being $\propto 2 \times$ larger than the corresponding one of the adsorbed ions. From a comparison of the theoretical to the experimental ARPES values, it can be concluded that the systems that yield a band-energy shift most compatible with the experiment are those in which $\mathrm{K}$ intercalates while $\mathrm{Li}$ adsorbs on the upper surface of $h \mathrm{BN}$, which is also in agreement with the angledependent XPS data. For these systems, the calculated $h \mathrm{BN}$ band shifts referred to the top of the $h \mathrm{BN} \pi$ band in the pristine $h \mathrm{BN} / \mathrm{Au}$ system are $1.80 \mathrm{eV}$ for $\mathrm{Li} / h \mathrm{BN} / \mathrm{Sub}$ and $2.73 \mathrm{eV}$ for $h \mathrm{BN} / \mathrm{K} / \mathrm{Sub}$. In Fig. 3, we report the atom-projected band structures calculated for the $2 \times 2$ unit cell. The energy shift of the $h \mathrm{BN} \pi$ band (black lines) in the functionalized systems is almost rigid and is experienced by all of the $h \mathrm{BN}$-derived bands. For the K case, the calculated value of the $h \mathrm{BN} \pi$-band shift $(2.73 \mathrm{eV})$ is in very good agreement with the ARPES data $(2.77 \mathrm{eV})$, thus indicating that the coverage considered in the model supercells is compatible with the one of the measured sample. In the case of $\mathrm{Li}$, the calculations overestimate the $h \mathrm{BN}$ band shift $(1.80 \mathrm{eV})$ with respect to the measured value $(0.9$ $\mathrm{eV}$ ). We attribute this to the following reason. To make the calculations feasible (smaller unit-cell size), we fixed the in-plane dopant superstructure to be $2 \times 2$ for both dopants. For the case of $\mathrm{K}$, this superstructure was experimentally verified for bulk $h \mathrm{BN}$ [11]. However, in the case of carbon materials, $\mathrm{Li}$ is known to form a $\sqrt{3} \times \sqrt{3}$ superstructure and nothing is known of its adsorption pattern on $h \mathrm{BN}$. Although 


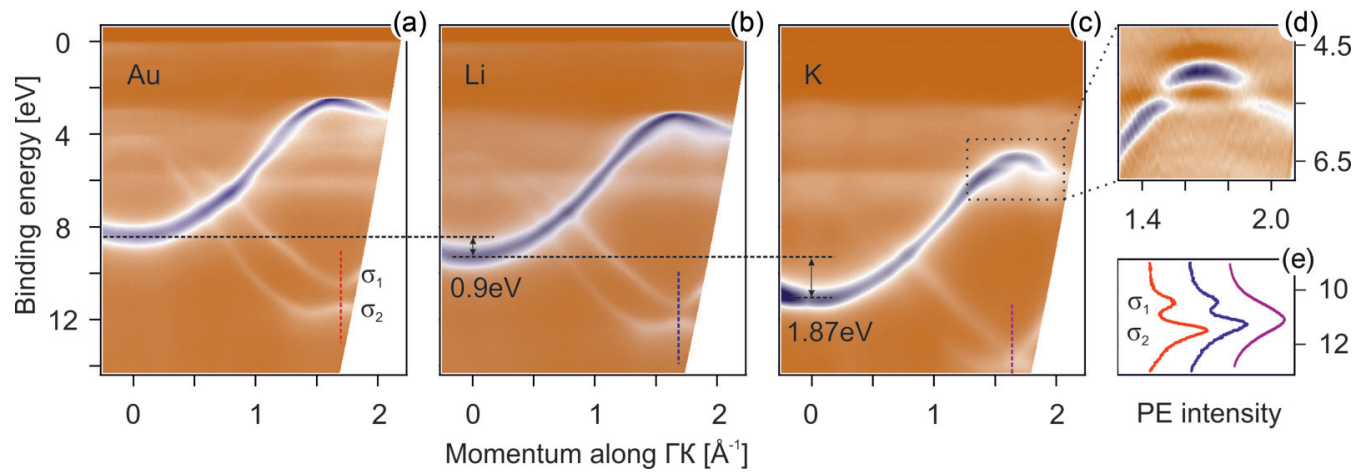

FIG. 2. (Color online) ARPES spectra along the $\Gamma K$ high-symmetry direction of the hexagonal BZ for (a) pristine $h \mathrm{BN}$ on $\mathrm{Au},(\mathrm{b}) \mathrm{Li}$ functionalized $h \mathrm{BN}$ on Au, and (c) K functionalized $h \mathrm{BN}$ on Au. The $K$ point is at $1.7 \AA^{-1}$. (d) A zoom of the $\pi$-band maximum for K-doped $h \mathrm{BN}$ close to the $K$ point. (e) Energy-dispersion curves through the $\sigma$ bands along the dotted lines. The Fermi-level position of the Au substrate is defined as zero.

we could not perform calculations with $\sqrt{3} \times \sqrt{3} \mathrm{Li}$ coverage, increasing the Li coverage to $1 / 4$ ML yielded larger shifts by $\sim 25 \%$. This therefore suggests that a better agreement with the experiment would be obtained with lower Li coverage, which therefore better represents the experimental conditions. Indeed, the saturation limit detected by ARPES for Li doping can originate from the fact that neutral $\mathrm{Li}$ clusters form that do not contribute to the charge-transfer reaction. Such clusters are equivalent to a diluted atomic Li coverage in the calculations. This assumption is strongly supported by the fact that we observed a $\mathrm{Li}^{0}$ component in the XPS spectrum at a dosage of about 1/4 ML (see Supplemental Material [26]). For this Li coverage, however, the ARPES-derived band structure was not affected. Since Li has a very small photoemission cross section and the resolution of XPS is limited, the signal from smaller quantities of partially ionized or neutral Li clusters (a) Li/hBN/Sub
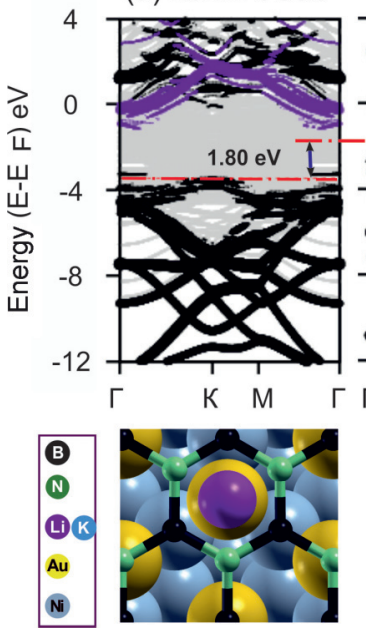

(b) $h \mathrm{BN} / \mathrm{Sub}$
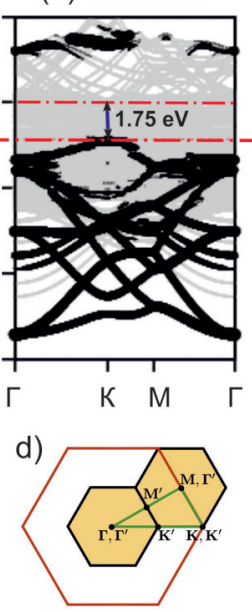

(c) $h \mathrm{BN} / \mathrm{K} / \mathrm{Sub}$
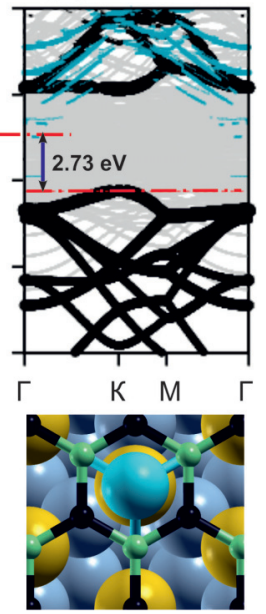

FIG. 3. (Color online) (a)-(c) DFT-calculated atom-projected band structures of alkali-metal functionalized $h \mathrm{BN}$ on $\mathrm{Au} / \mathrm{Ni}(\mathrm{Ni}$ : gray; $h \mathrm{BN}$ : black; Li: purple; K: blue). The thickness of the lines varies according to the contribution of each atomic state at that particular energy. A sketch representing the top view of the respective functionalized systems is shown in the bottom panel of (a) and (c). (d) Folding of the $2 \times 2 \mathrm{BZ}$ with respect to the $1 \times 1$ zone. can be undetectable. In summary, the observed shift for Li qualitatively agrees with the experiment in both cases and is in very good quantitative agreement with the case of $K$.

We now move to the analysis of the optimized geometry and to the charge distribution resulting from the DFT calculations. The bottom panel of Fig. 3 displays the top view of the simulated unit cells. The side view is shown in the inset of Fig. 4. Alkali functionalization induces structural distortions and relaxations to the reference $h \mathrm{BN} / \mathrm{Au}$ lattices. In the case of $\mathrm{K}$, the distance between the metal substrate and the $h \mathrm{BN}$ sheet increases from $3.05 \AA$ to $5.41 \AA$. In this configuration, the plane of the $\mathrm{K}$ atoms is $2.53 \AA$ above the metal and $2.88 \AA$ below the $h \mathrm{BN}$ sheet, while in the case of $\mathrm{Li}$, the dopants are predicted to be $1.90 \AA$ above the $h \mathrm{BN}$ sheet. In addition, this dopant induces an out-of-plane distortion of the $h \mathrm{BN}$ honeycomb lattice, with the $\mathrm{N}$ and $\mathrm{B}$ atoms relaxing outwards and inwards, respectively, resulting in a corrugation of $0.25 \AA$. The corrugation of the $h \mathrm{BN}$ lattice predicted for the $\mathrm{Li} / h \mathrm{BN} / \mathrm{Sub}$ system resembles the polarization of an ionic honeycomb lattice subjected to a transverse electric field. It is due to the polar character of the B-N bond that leads to partial negative/positive excess charges on the N/B atoms. Conversely, the fact that a negligible lattice corrugation of $0.04 \AA$ is predicted for the $h \mathrm{BN} / \mathrm{K} / \mathrm{Sub}$ case suggests that the $h \mathrm{BN}$ sheet is exposed to a very small effective transverse electric field when the dopant is below the $h \mathrm{BN}$ plane. In addition, the rigid shift of the $h \mathrm{BN} \pi$ band is also consistent with a dopant-driven electric field at the interface. The following analysis of the potential across the interface allows us to identify these effective fields that modify the structural and electronic properties of the functionalized $h \mathrm{BN} / \mathrm{Au}$ systems.

Starting from the Li case, we report in Fig. 4 the planar average of the total Kohn-Sham (KS) potential along the $z$ direction of the $\mathrm{Li} / h \mathrm{BN} / \mathrm{Sub}$ supercell, $V(z)$ (red line). For further analysis, we construct a model reference substrate from the $\mathrm{Li} / h \mathrm{BN} / \mathrm{Sub}$ supercell by removing the $\mathrm{Li}$ atom, while leaving the coordinates of all other atoms unaltered. From here onwards, we refer to this auxiliary system as $h \mathrm{BN} / \mathrm{Sub}^{\star}$ and notate the corresponding potential $V_{\mathrm{BN} / \mathrm{Sub}^{\star}}$ as $V^{\star}(z)$ in Fig. 4 (blue line). The average potential in the $\mathrm{Ni}$ slab is taken as the zero reference for $V(z)$. The difference $\Delta V(z)=V(z)-$ $V^{\star}(z)$ is an approximation for the effective potential acting 
(a) $\mathrm{Li} / h \mathrm{BN} / \mathrm{Sub}$

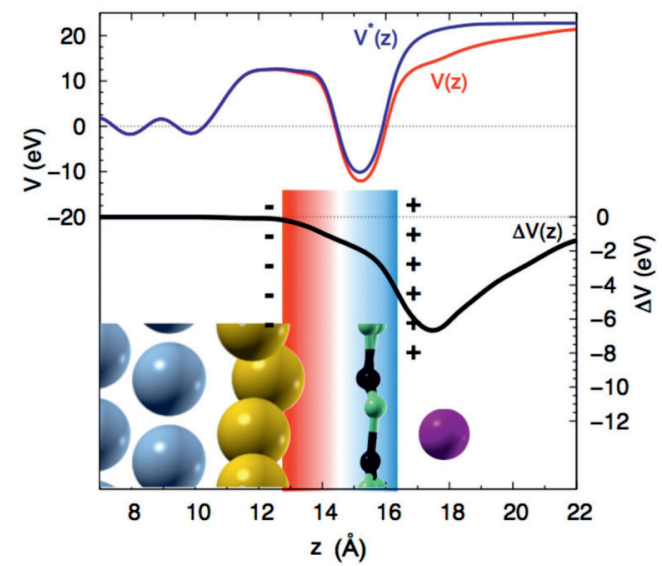

(b) $h \mathrm{BN} / \mathrm{K} / \mathrm{Sub}$

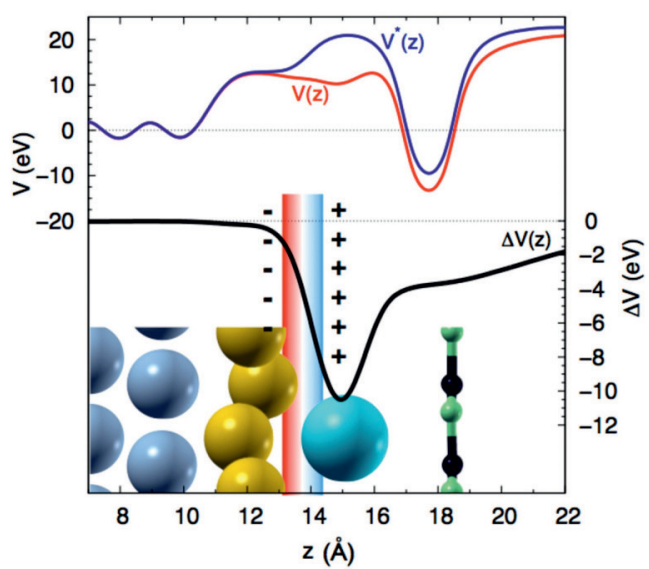

FIG. 4. (Color online) DFT-calculated planar average of the total Kohn-Sham potential along the $z$ direction of the supercell plotted for (a) $\mathrm{Li} / h \mathrm{BN} / \mathrm{Sub}$ and (b) $h \mathrm{BN} / \mathrm{K} / \mathrm{Sub}$. The blue $\left[V^{\star}(z)\right]$ and red $[V(z)]$ lines shows the respective potential of the auxiliary substrate and the functionalized system (see text for the details of the notation). $\Delta V(z)$ represents the effective potential due to the presence of the substrate. The capacitor is indicated by a box with red-blue gradient.

on the system due to the dopant (black line in Fig. 4). $\Delta V$ is zero in the region of the metal support, while there is a drop between the $\mathrm{Au}$ and $\mathrm{Li}$ planes, leading to an effective potential of $1.95 \mathrm{eV}$ at the $h \mathrm{BN}$ plane. This additional potential is responsible for the energy shift of the $h \mathrm{BN} \pi$ bands due to Li. There is, however, a small discrepancy between the value of this shift $(1.95 \mathrm{eV})$ and the corresponding one resulting from the band structure calculation $(1.80 \mathrm{eV}$; see Fig. 3$)$. This originates from the different reference substrates that are used in the two analyses, $h \mathrm{BN} / \mathrm{Sub}^{\star}$ and $h \mathrm{BN} / \mathrm{Sub}^{1}$

Most interestingly, the above analysis shows that the $h \mathrm{BN}$ plane is within a capacitorlike linear potential profile along the $z$ direction [Fig. 4(a)]. Indeed, the binding of Li above the $h \mathrm{BN}$ sheet drives a charge reorganization, giving rise to $\delta^{+}$and $\delta^{-}$ net charges at the $\mathrm{Li}$ and Au layers, respectively. This charge redistribution effectively generates an interface capacitor and a related effective field, which embeds the dielectric $h \mathrm{BN}$ plane, and therefore rigidly shifts its $\pi$ bands. Quite consistently, the ionic $h \mathrm{BN}$ lattice responds to this effective field by a polarized structural distortion, i.e., the lattice corrugation described above.

The same analysis applied to the K case [Fig. 4(b)] shows the presence of an effective capacitor at the $\mathrm{Au} / \mathrm{K}$ interface. The important difference between the two cases is the position of the $h \mathrm{BN}$ plane with respect to this capacitor: inside the interfacial capacitor in the case of $\mathrm{Li}$ and outside it in the case of K. As such, the $h \mathrm{BN}$ is not directly subjected to an effective field in the $h \mathrm{BN} / \mathrm{K} / \mathrm{Sub}$ system and therefore the effective potential profile $(\Delta V)$ at the $h \mathrm{BN}$ plane is essentially constant. The interface effective field and the related capacitor introduce a potential difference at the $h \mathrm{BN}$ plane with respect

\footnotetext{
${ }^{1}$ The use of the model $h \mathrm{BN} / \mathrm{Sub}^{\star}$ supercell has numerical and physical justifications. It enables us not only to precisely map the charge reorganization, but also to disentangle the electronic contribution from the structural one.
}

to the reference substrate. This potential difference drives the energy shift of the $h \mathrm{BN} \pi$ bands for the K case. We note that the potential difference $\Delta V$ at the $h \mathrm{BN}$ plane reported in Fig. 4 $(3.76 \mathrm{eV})$ for the $\mathrm{K}$ case is larger than the energy shift obtained with the band structure calculation $(2.73 \mathrm{eV})$. This is because the $\Delta V$ analysis accounts only for the electronic contribution, i.e., the electron redistribution due to $\mathrm{K}$ insertion below the $h \mathrm{BN}$ plane. The structural relaxation that reduces the $h \mathrm{BN}-\mathrm{Sub}$ distance from $5.41 \AA$ (as in the auxiliary $h \mathrm{BN} / \mathrm{Sub}^{\star}$ supercell) to $3.05 \AA$ (as in the fully relaxed, pristine $h \mathrm{BN} / \mathrm{Sub}$ supercell) causes an additional potential difference at the $h \mathrm{BN}$ plane that brings the total value of the calculated $\Delta V$ to $2.65 \mathrm{eV}$, consistent with the band structure result (see Appendix for details).

\section{CONCLUSIONS}

In conclusion, we have performed in situ ionic functionalization of the $h \mathrm{BN} / \mathrm{Au}$ interface. Systems prepared in this way have large single-crystalline domain sizes and do not suffer from the inherent disadvantages regarding size of nanofabricated samples for electronic gating. The effects of gating and electric fields were achieved by intercalation of $\mathrm{K}$ in between the $h \mathrm{BN}$ and the metal substrate and by deposition of Li onto $h \mathrm{BN}$. We have studied the effects of gating and electric fields using the ARPES method, where we could directly investigate the effect on the electron energy-dispersion relations. DFT simulations rationalize the measured near-rigid shift of the $h \mathrm{BN}$ bands in terms of interfacial electric fields and potential differences between the metallic sample and the supported $h \mathrm{BN}$ monolayer. The origin of these fields is the ionization of the alkali atoms that lead to formation of the positively charged layers that could be located either at the metal substrate (for the case of $\mathrm{K}$ ) or above the $h \mathrm{BN}$ plane (for the case of $\mathrm{Li}$ ).

Our findings indicate that the electronic levels of $h \mathrm{BN}$ monolayers can be controlled by alkali-metal doping, thus 
suggesting that the $h \mathrm{BN} /$ metal interface can indeed be functionalized, opening the way for its potential applications as an active element in electronic devices. One example could be a vertical tunneling diode where $h \mathrm{BN}$ is sandwiched in between $\mathrm{Au}$ and $\mathrm{Li}$. Due to the inherent electric field, we expect anisotropic transport characteristics between a contact on top of a Li-doped $h \mathrm{BN}$ sheet and a metallic substrate.

\section{ACKNOWLEDGMENTS}

The research leading to these results has received funding from the European Community FP7 CALIPSO (Grant No. 312284) Transnational Access Program. A.G. and N.V. acknowledge funding from the NanoCF project. A.G. acknowledges the ERC Grant No. 648589 - SUPER-2D. N.V. acknowledges the Russian Science Foundation (Grant No. 14-13-00747), the Ministry of Education and Science of the Russian Federation (Grant No. 14.585.21.0004), and the Austrian Science Fund (FWF), Project No. I 377-N16, Theory and spectroscopy on functionalized graphene. We thank HZB for the allocation of synchrotron radiation beam time. A.N. and C.W. acknowledge funding from the Science and Technology of Nanosystems programme (Project No. 431103Molecular Building Blocks/Supramolecular Networks). S.F. and S.P. acknowledge funding from MIUR PRIN, Grant No. 20105ZZTSE. D.U. and D.V. acknowledge Saint-Petersburg State University for research Grant No. 11.37.634.2013 and BMBF (Grant No. 05K12OD3). D.U. and A.F. acknowledge RFBR (Grant No. 14-02-31150).

\section{APPENDIX: EXPERIMENTAL AND COMPUTATIONAL DETAILS}

\section{Experimental details}

The sample synthesis was done via chemical-vapor deposition of borazine [19-21,27] on a Ni(111) film on a W(110) crystal. This was followed by $\mathrm{Au}$ intercalation [21] and evaporation of $\sim 1$ monolayer of alkali metals (in respect to the bulk fcc $\mathrm{K}$ or Li structure) from SAES getters onto the $h \mathrm{BN}$ surface controlled by quartz microbalance and subsequent annealing to $400{ }^{\circ} \mathrm{C}$ for $5 \mathrm{~min}$. Freshly prepared samples were checked via the low-energy electron diffraction (LEED) technique. The well-known LEED reflexes exhibited the moiré $9 \times 9$ structure, like for graphene on $\mathrm{Au}$ [9]. Since no additional LEED spots were detected, we concluded that $\mathrm{Li}$ and $\mathrm{K}$ do not form regular structures in these conditions, similar to what was also found for the graphene/Au interface before $[8,24]$. The dopant concentrations were also estimated by XPS, including inelastic scattering inside $h \mathrm{BN}$ for the K case, and were found to be consistent with quartz microbalance measurements within an error bar of about $5 \%$.

The ARPES experiments were performed using the photoelectron spectrometer at the IFW-Dresden equipped with a Scienta SES-200 hemispherical electron energy analyzer and a high-flux He resonance lamp (Gammadata VUV-5010) in combination with a grating monochromator. All ARPES spectra were acquired at room temperature and a photon energy of $40.8 \mathrm{eV}(\mathrm{He} \mathrm{II} \alpha)$, with an angular resolution of $0.2^{\circ}$ and a total-energy resolution of $50 \mathrm{meV}$. Electron band dispersions were measured along the $\Gamma K M$ direction of the BZ by varying the polar-emission angle. The XPS and NEXAFS experiments on ML- $h \mathrm{BN}$ were carried out using linearly polarized radiation at the HESGM and RGBL beam lines at the Helmholtz-Zentrum Berlin. The Au4 $f$ line was used to calibrate the line positions of the core-level measurements.

\section{Computational details}

We performed atomistic DFT simulations using the PerdewBurke-Ernzerhof (PBE) exchange-correlation functional, employing the plane-wave ultrasoft pseudopotential method, as implemented in the PWscf code of the QUANTUM ESPRESSO distribution. The plane-wave basis set and density representation were truncated by energy cutoff of 35 and $350 \mathrm{Ry}$, respectively. Van der Waals dispersion corrections [28] as well as spin polarization were considered explicitly for all of the calculations. We modeled the substrate by adsorbing a 3/4 ML of Au atoms on the top, fcc, and hpc sites of the five-layered $\mathrm{Ni}(111) 2 \times 2$ supercell surface. A $2 \times 2$ pristine $h \mathrm{BN}$ is deposited on the substrate by aligning the $\mathrm{B}$ atoms on the top and $\mathrm{N}$ atoms on the fcc sites with respect to the $\mathrm{Ni}(111)$ surface. The $h \mathrm{BN}$ lattice constant was stretched to match with the $\mathrm{Ni}(111)$ lattice. In these $2 \times 2$ supercells, one $\mathrm{K} / \mathrm{Li}$ (single adatom) was adsorbed above/below at the hollow site of the $h$ BN Sub lattice.

For a direct evaluation of the potential difference $\Delta V$, it is crucial here to have an auxiliary supercell, analogous to the one defined above for the Li case. The actual value of the $\Delta V$ at the $h \mathrm{BN}$ plane obtained by subtracting the potential of the pristine $h \mathrm{BN} / \mathrm{Au}$ supercell from the one of the $h \mathrm{BN} / \mathrm{K} / \mathrm{Au}$ system is $2.65 \mathrm{eV}$, which is quite consistent with the energy shift of the $h \mathrm{BN} \pi$ band in the calculated band structure. However, this subtraction does not allow one to obtain a meaningful effective potential profile resulting from the charge redistribution upon $\mathrm{K}$ insertion below the $h \mathrm{BN}$ plane. In this subtraction, the deep potential minima of the $\mathrm{B}$ and $\mathrm{N}$ nuclei do not cancel out because the $h \mathrm{BN}$ sheet is located at different positions in the two supercells $(h \mathrm{BN} / \mathrm{K} / \mathrm{Au}$ and $h \mathrm{BN} / \mathrm{Au})$; hence the need of using the auxiliary supercell, which is obtained by removing the $\mathrm{K}$ atom from the $h \mathrm{BN} / \mathrm{K} / \mathrm{Au}$ supercell while leaving the coordinates of all other atoms unaltered. This $h \mathrm{BN} / \mathrm{K} / \mathrm{Au}$ supercell, in which the $h \mathrm{BN}$ sheet has the same coordinate as in $h \mathrm{BN} / \mathrm{K} / \mathrm{Au}$, allows one to extract the physically meaningful effective potential profile resulting from the electron redistribution. The actual value of the $\Delta V$ at the $h \mathrm{BN}$ plane obtained from this analysis, 3.76 $\mathrm{eV}$ [see Fig. 4(b)], is referred to the auxiliary supercell used in the subtraction, and is obviously overestimated because of the larger distance of the $h \mathrm{BN}$ plane from the substrate in the reference system. This large energy shift accounts only for the electronic contribution. As in the Li case, the energy shift due to the structural relaxation (the $h \mathrm{BN}$ sheet relaxing closer to the metal substrate as in the $h \mathrm{BN} / \mathrm{Au}$ reference) causes an additional potential difference at the $h \mathrm{BN}$ plane that brings the total value of the calculated $\Delta V$ to $2.65 \mathrm{eV}$, consistent with the band structure result. 
[1] H. Dil, J. Lobo-Checa, R. Laskowski, P. Blaha, S. Berner, J. Osterwalder, and T. Greber, Surface trapping of atoms and molecules with dipole rings, Science 319, 1824 (2008).

[2] K. Watanabe, T. Taniguchi, T. Niiyama, K. Miya, and M. Taniguchi, Far-ultraviolet plane-emission hand-held device based on hexagonal boron nitride, Nat. Photon. 3, 591 (2009).

[3] K. Watanabe, T. Taniguchi, and H. Kanda, Direct-bandgap properties and evidence for ultraviolet lasing of hexagonal boron nitride single crystal, Nat. Mater. 3, 404 (2004).

[4] G. Gao, W. Gao, E. Cannuccia, J. Taha-Tijerina, L. Balicas, A. Mathkar, T. N. Narayanan, Z. Liu, B. K. Gupta, J. Peng et al., Artificially stacked atomic layers: Toward new van der Waals solids, Nano Lett. 12, 3518 (2012).

[5] L. Britnell, R. V. Gorbachev, R. Jalil, B. D. Belle, F. Schedin, A. Mishchenko, T. Georgiou, M. I. Katsnelson, L. Eaves, S. V. Morozov et al., Field-effect tunneling transistor based on vertical graphene heterostructures, Science 335, 947 (2012).

[6] P. Jaffrennou, J. Barjon, T. Schmid, L. Museur, A. Kanaev, J.-S. Lauret, C. Y. Zhi, C. Tang, Y. Bando, D. Golberg, B. AttalTretout, F. Ducastelle, and A. Loiseau, Near-band-edge recombinations in multiwalled boron nitride nanotubes: Cathodoluminescence and photoluminescence spectroscopy measurements, Phys. Rev. B 77, 235422 (2008).

[7] A. Rubio, J. L. Corkill, and M. L. Cohen, Theory of graphitic boron nitride nanotubes, Phys. Rev. B 49, 5081(R) (1994).

[8] D. Haberer, L. Petaccia, A. V. Fedorov, C. S. Praveen, S. Fabris, S. Piccinin, O. Vilkov, D. V. Vyalikh, A. Preobrajenski, N. I. Verbitskiy, H. Shiozawa, J. Fink, M. Knupfer, B. Büchner, and A. Grünels, Anisotropic Eliashberg function and electronphonon coupling in doped graphene, Phys. Rev. B 88, 081401(R) (2013).

[9] D. Haberer, D. V. Vyalikh, S. Taioli, B. Dora, M. Farjam, J. Fink, D. Marchenko, T. Pichler, K. Ziegler, S. Simonucci et al., Tunable bandgap in hydrogenated quasi-free-standing graphene, Nano Lett. 10, 3360 (2010).

[10] D. Usachov, O. Vilkov, A. Grüneis, D. Haberer, A. Fedorov, V. K. Adamchuk, A. B. Preobrajenski, P. Dudin, A. Barinov, M. Oehzelt et al., Nitrogen-doped graphene: Efficient growth, structure, and electronic properties, Nano Lett. 11, 5401 (2011).

[11] G. L. Doll, J. S. Speck, G. Dresselhaus, M. Dresselhaus, K. Nakamura, and S. Tanuma, Intercalation of hexagonal boron nitride with potassium, J. Appl. Phys. 66, 2554 (1989).

[12] G. Giovannetti, P. A. Khomyakov, G. Brocks, P. J. Kelly, and J. van den Brink, Substrate-induced band gap in graphene on hexagonal boron nitride: $A b$ initio density functional calculations, Phys. Rev. B 76, 073103 (2007).

[13] J. Xue, J. Sanchez-Yamagishi, D. Bulmash, P. Jacquod, A. Deshpande, K. Watanabe, T. Taniguchi, P. Jarillo-Herrero, and B. J. LeRoy, Scanning tunneling microscopy and spectroscopy of ultra-flat graphene on hexagonal boron nitride, Nat. Mater. 10, 282 (2011).

[14] K. H. Khoo, M. S. C. Mazzoni, and S. G. Louie, Tuning the electronic properties of boron nitride nanotubes with transverse electric fields: A giant dc Stark effect, Phys. Rev. B 69, 201401(R) (2004).

[15] C.-H. Park and S. G. Louie, Energy gaps and stark effect in boron nitride nanoribbons, Nano Lett. 8, 2200 (2008).

[16] M. Ishigami, J. D. Sau, S. Aloni, M. L. Cohen, and A. Zettl, Observation Of the Giant Stark Effect in Boron-Nitride Nanotubes, Phys. Rev. Lett. 94, 056804 (2005).

[17] C. Attaccalite, L. Wirtz, A. Marini, and A. Rubio, Efficient gatetunable light-emitting device made of defective boron nitride nanotubes: From ultraviolet to the visible, Sci. Rep. 3, 2698 (2013).

[18] M. Radosavljević, J. Appenzeller, V. Derycke, R. Martel, P. Avouris, A. Loiseau, J.-L. Cochon, and D. Pigache, Electrical properties and transport in boron nitride nanotubes, Appl. Phys. Lett. 82, 4131 (2003).

[19] C. Oshima and A. Nagashima, Ultra-thin epitaxial films of graphite and hexagonal boron nitride on solid surfaces, J. Phys.: Condens. Matter 9, 1 (1997).

[20] A. B. Preobrajenski, M. L. Ng, N. A. Vinogradov, A. S. Vinogradov, E. Lundgren, A. Mikkelsen, and N. Martensson, Impact of oxygen coadsorption on intercalation of cobalt under the h-BN nanomesh, Nano Lett. 9, 2780 (2009).

[21] D. Usachov, V. K. Adamchuk, D. Haberer, A. Grüneis, H. Sachdev, A. B. Preobrajenski, C. Laubschat, and D. V. Vyalikh, Quasifreestanding single-layer hexagonal boron nitride as a substrate for graphene synthesis, Phys. Rev. B 82, 075415 (2010).

[22] M. Corso, W. Auwärter, M. Muntwiler, A. Tamai, T. Greber, and J. Osterwalder, Boron nitride nanomesh, Science 303, 217 (2004).

[23] F. Mahvash, E. Paradis, D. Drouin, T. Szkopek, and M. Siaj, Space-charge limited transport in large-area monolayer hexagonal boron nitride, Nano Lett. 15, 2263 (2015).

[24] A. V. Fedorov, N. I. Verbitskiy, D. Haberer, C. Struzzi, L. Petaccia, D. Usachov, O. Y. Vilkov, D. V. Vyalikh, J. Fink, M. Knupfer et al., Observation of a universal donordependent vibrational mode in graphene, Nat. Commun. 5, 4257 (2014).

[25] A. B. Preobrajenski, S. A. Krasnikov, A. S. Vinogradov, M. L. Ng, T. Käämbre, A. A. Cafolla, and N. Mårtensson, Adsorptioninduced gap states of h-BN on metal surfaces, Phys. Rev. B 77, 085421 (2008).

[26] See Supplemental Material at http://link.aps.org/supplemental/ 10.1103/PhysRevB.92.125440 for the additional XPS data for doped hBN monolayer by $1 / 4 \mathrm{ML}$ of $\mathrm{Li}$.

[27] Y. Shi, C. Hamsen, X. Jia, K. K. Kim, A. Reina, M. Hofmann, A. L. Hsu, K. Zhang, H. Li, Z.-Y. Juang et al., Synthesis of few-layer hexagonal boron nitride thin film by chemical vapor deposition, Nano Lett. 10, 4134 (2010).

[28] S. Grimme, Semiempirical GGA-type density functional constructed with a long-range dispersion correction, J. Comp. Chem. 27, 1787 (2006). 